\section{Aspirin resistance}

\section{Opinion}

In the pathogenesis of vascular cerebral insufficiency, a significant place is occupied by atherosclerotic lesions of the vascular wall, as well as their combination with disorders of coagulation and anticoagulation systems. Intravascular macro and micro-coagulation results in the formation of a blood clot and cerebrovascular accident. Maintenance of platelet and plasma coagulation hemostasis at the physiological level for a long time helps to postpone or delay the development of vascular complications. In addition, the peculiarities of the course of acute cerebral ischemia are largely determined by the pre-existing chronic cerebral ischemia and the state of its metabolism and imbalance in the hemostasis system. Therefore, the importance of correction of pathological changes in the system of hemorrhage and vascular endothelium is undeniable and necessitates the maintenance of the antithrombotic potential of the vascular wall under conditions of before stroke forms of cerebrovascular pathology.

To date, the most common, relatively safe and affordable drug that has not lost its status as an antiplatelet reference standard with more than 100years of experience in medicine is acetylsalicylic acid (ASA) aspirin. The effectiveness of low doses of ASA (75-150mg/ day) for the secondary prevention of cardiovascular disease has been demonstrated in numerous randomized trials (about 100). At the same time, studies have shown that ASA therapy only warns about $25 \%$ of adverse cardiovascular events. In addition, with the use of various laboratory methods for evaluating the function of platelets, data on a changing and even paradoxical response to the drug have been accumulated, both in healthy people and in patients with atherosclerotic lesions.

With the introduction into the clinic of diagnostic methods of plasma coagulation hemostasis and platelet aggregation activity on various inductors, it was possible to adequately assess the effectiveness of antiplatelet therapy. Patients taking antiplatelet agents may have a broad variation in response, which is consistent with normal distribution. Clinical implications of this variability are potentially important because they are associated with a high risk of thrombotic events in patients with low antiagregant sensitivity and an increased risk of bleeding with a pronounced response. According to the results of multicenter studies (Medlsne, Embase, CHINAHL) in $20 \%$ of patients who use ASA for prophylactic purposes, there is a paradoxical progregant effect. The study of this phenomenon led to the emergence of such a concept as "aspirin resistance". Resistance to ASA-a phenomenon quite common and occurs, according to various data, with a frequency from $5 \%$ to $48 \%$.

At the moment, many causes of aspirin resistance are discussed. According to the memorandum of the Working Group on the Resistance Study to the ASA of the International Society for
Volume 2 Issue 3 - 2018

\author{
Mykhalojko Ja I \\ Ivano-Frankivsk National Medical University, Ukraine
}

Correspondence: Mykhalojko Ja I, Ivano-Frankivsk National Medical University, Ukraine, Email myhalojko@i.ua

Received: May 01, 2018| Published: May 24, 2018

Thrombosis and Hemostasis, it may be due to: an increase in the formation of metabolites of arachidonic acid by lipoxygenase, which leads to suppression of the synthesis of prostacyclin in the endothelial cells, despite the decrease in the production of thromboxane A2; poor patient's commitment to treatment with aspirin (in $2 \%$ of patients) or inadequate dosage; the polymorphism of the cyclooxygenase gene or GP IIb/IIIa glycoprotein gene, which causes the synthesis of glycoproteins with high affinity for fibrinogen; endothelial dysfunction, which is accompanied by an increase in the activity of the Villebrand factor and a decrease in the level of prostacyclin; hypercholesterolemia, which increases the rigidity of platelet membranes and worsens the sensitivity of glycoprotein receptors.

However, according to a number of scientific papers, there are reasons that can lead to the development of secondary insensitivity to aspirin, which is even more dangerous than the primary, since it is difficult to predict and diagnose its development in a timely manner. However, regardless of the cause of resistance, an inadequate response to ASA is associated with an increase in the risk of cardiovascular complications by four times as compared with aspirin-sensitive patients, which necessitates a deeper study of the causes of AR, its availability and ways to optimize anti-aggregant therapy in prevention vascular catastrophes. The only possibility to diagnose existing resistance to aspirin-both primary and secondary-is the regular control of the level of aggregation of platelet activity.

One of the possible ways to overcome the platelet resistance is the use of antiplatelet drugs (or their combination) with other mechanisms of action. Alternative antiplatelet drugs in the case of AR that have a different point of application may be thienopyridines-blockers of aP2Y12 receptors. These include: clopidogrel, ticlopidine, prasugrel.

\section{Acknowledgements}

None.

\section{Conflict of interest}

There is no conflict of interest. 